\title{
A Flexible Method for Localisation and Classification of Footprints of Small Species
}

\author{
Haokun Geng ${ }^{1}$, James Russell ${ }^{2}$, Bok-Suk Shin ${ }^{1}$, \\ Radu Nicolescu ${ }^{1}$, and Reinhard Klette ${ }^{1}$ \\ 1 Department of Computer Science, University of Auckland, Auckland, New Zealand \\ hgen001@aucklanduni.ac.nz, \{b.shin,r.nicolescu,r.klette\}@auckland.ac.nz \\ 2 School of Biological Sciences, Department of Statistics, University of Auckland, \\ Auckland, New Zealand \\ j.russell@auckland.ac.nz
}

\begin{abstract}
In environmental surveillance, ecology experts use a standard tracking tunnel system to acquire tracks or footprints of small animals, so that they can measure the presence of any selected animals or detect threatened species based on the manual analysis of gathered tracks. Unfortunately, distinguishing morphologically similar species through analysing their footprints is extremely difficult, and even very experienced experts find it hard to provide reliable results on footprint identification. This expensive task also requires a great amount of efforts on observation. In recent years, image processing technology has become a model example for applying computer science technology to many other study areas or industries, in order to improve accuracy, productivity, and reliability. In this paper, we propose a method based on image processing technology, it firstly detects significant interest points from input tracking card images. Secondly, it filters irrelevant interest points in order to extract regions of interest. Thirdly, it gathers useful information of footprint geometric features, such as angles, areas, distance, and so on. These geometric features can be generally found in footprints of small species. Analysing the detected features statistically can certainly provide strong proof of footprint localization and classification results. We also present experimental results on extracted footprints by the proposed method.With appropriate developments or modifications, this method has great potential for applying automated identification to any species.
\end{abstract}

\section{Introduction}

Computer-based systems have been a common technique of humankind to perform activities that have to be repeated numerous times [5]. Identifying small species from their footprints is one of such activities. Currently ecological experts need to spend much effort and time identifying footprints from inked tracking cards, highly dependent on the experts' knowledge and experiences, and the manual identification analysis often needs to be repeated on many tracking cards.

Therefore, we would like to initialise a work based on many previously researched theoretical findings, with knowledge from ecology, especially from the 


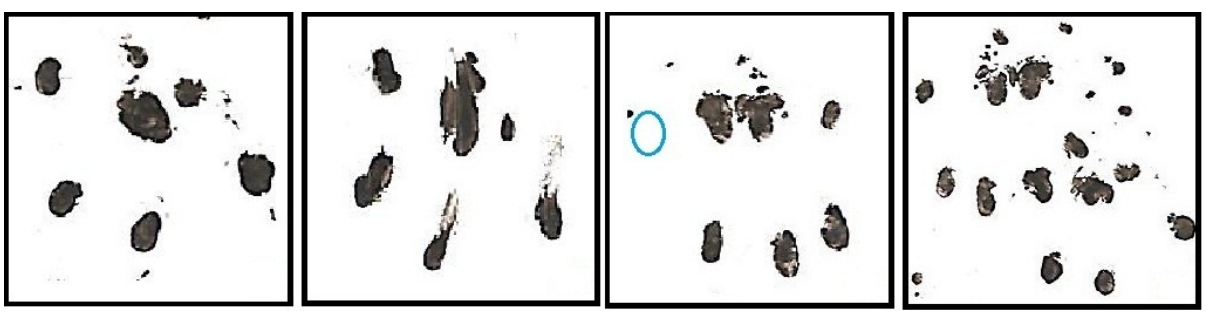

Fig. 1. Samples of mice footprints in different situations. From left to right: Normal front footprint, sliding front footprint, missing toe hind footprint, and overlapped hind\&front footprints

study area of track recognition, and to transfer them into a practical technique to assist ecological experts in analysing inked tracking cards.

The demand for such systems that can process the automated identification of species from their scanned footprint images is most likely to increase in the future 8 . It becomes essential to have a working application that can be properly incorporated into the current system, handles the repetitive jobs, and outputs accurate and reliable results.

However, the presentations of footprints are varied, the 'puzzle' is that the images of a footprint may have very different appearances (as shown in Fig 1). Besides normal footprints, other undesirable image data include sliding footprints, missing toe footprints, and overlapped footprints. A single tracking card may contain footprints from $\geq 1$ individuals.

Before any further analysis can be carried out by the automated recognition algorithm, those varied representations of the footprints need to be transformed into digitalised geometric models. Correctly understanding and handling the transformation process is certainly a difficult task.

In this paper, we will firstly introduce the current standard track acquisition procedure. Then we will describe how a footprint can be digitalised and understood by our image processing application. This involves a rule based footprint recognition algorithm that performs automated footprint localisation and classification. Additionally, we would like to present the conceptualization of an integrated proposed system, and suggest some possible future work.

\section{Track Acquisition of Small Species}

The Tracking Tunnel System is a widely used standard procedure for collecting tracks of small animals to gain an index of the abundance of target small species in New Zealand [2]. It is a cost-effective method to collect tracks of small species over large areas [10]. Providing reasonable analysis and reliable results on the estimate of species' presence plays an important role in ecological research when ecologists decide to study rare species or assess community composition for environmental surveillance or pest control [7]. 


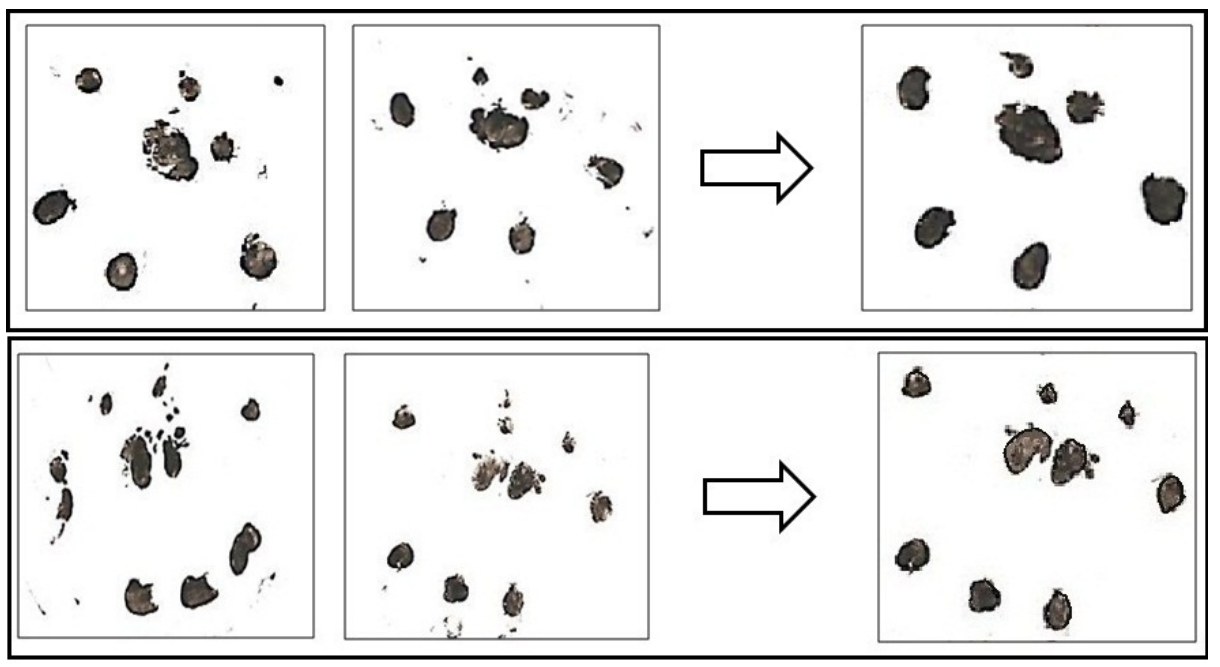

Fig. 2. Top: Isolated front footprint model example. Bottom: Isolated hind footprint model example.

Traditionally, tracks or footprints are collected by this tracking tunnel system, and the identification of tracks and footprints is handled manually by experienced wildlife experts 3 . The basic principle of animal tracking is firstly to recognise single footprints from a number of unknown footprints, and then to identify the species based on the analysis of its footprints [10.

The tracking tunnel system is considered the first step when ecologists would like to non-invasively monitor or study a selected species. The collected tracks or footprints need to be analysed manually by human experts. In the identification procedure, distinguishing among many morphologically similar species through analysing their footprints is extremely difficult, and one single tracking card can also contain footprints from different species [10]. Our method aims to ultimately implement an automated recognition process to assist experts in the current identification procedure.

\section{Footprint Geometric Analysis}

The further implementation of the track recognition algorithm would highly depend on the understanding of the footprint geometric models of targeted species. In the following experiments, we choose house mice (Mus musculus) as our major object of study.

We isolate normal footprints from many tracks on a tracking card. The front foot for a house mouse usually has four toes, the hind foot usually has five toes [10]. The toes of the front foot are evenly distributed around the central pad. The hind foot normally has three toes bunched in front of the central pad that can roughly form a straight line. Based on the previous studies [10] and our experimental set of tracking cards, we isolated normal footprints from tracks. 

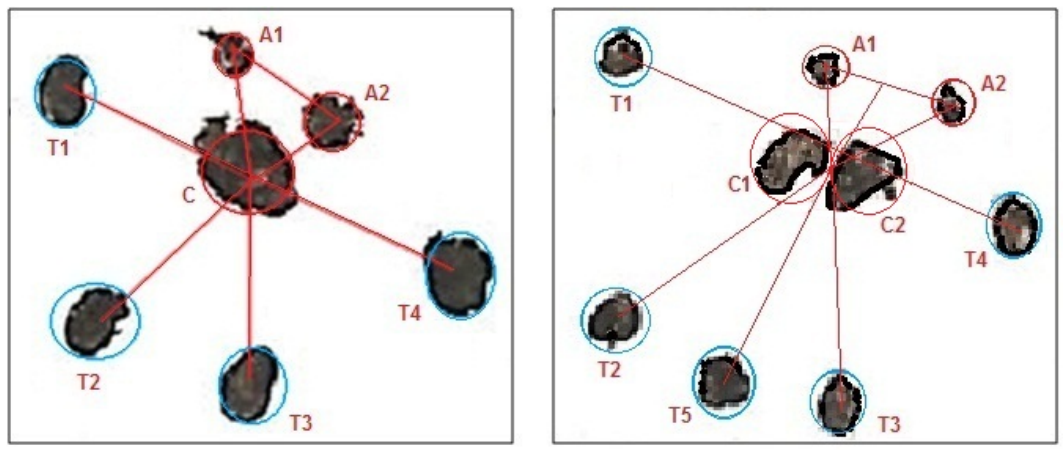

Fig. 3. Left: Geometric model for mice front footprint. Right: Geometric model for mice hind footprint.

The isolated front footprint model is shown in Figure 2 (top), and the isolated hind footprint model is shown in Figure 2 (bottom).

Manually analysing the relations among toes and central pads of the isolated footprints is an essential step for footprint localisation and classification (i.e., front or hind footprints). Figure 3 visually represents how geometric features will be analysed by the proposed algorithm.

Figure 3 (left) is an isolated front footprint, it has a clear geometric structure: a central pad is in the centre of the footprint. There are two accessorial pads and four toes evenly distributed around the central pad. In most cases, the central pad has the larger size than other nodes in this particular region. The toe prints are marked by blue circles, the central pad and accessorial pads prints are marked by red circles. The central pad is distributed in the middle point of that line segment. The central pad and two accessorial pads clearly form a triangle. Also there are three straight lines intersecting the central pad, they are $\overline{T_{1} T_{4}}, \overline{T_{2} A_{2}}$, and $\overline{T_{3} A_{1}}$.

Figure 3 (right) is an isolated hind footprint, it has a similar geometric structure to the front footprint. However, by contrast it has a differently formed central pad which consists of two vice pads. It also has three toes in the front, they can roughly form a straight line $\overline{T_{2} T_{3}}$ that is approximately parallel with the line formed by the two outer toes $\overline{T_{1} T_{4}}$. Comparing with the front footprint, the formation of the hind central pads and the number of toes could be two significant conditions of front and hind footprints classification.

\section{Footprint Extraction}

We propose a method for extracting regions of interest using improved OpenSurf libraries 1 with the rule-based conditional filtering and geometric model. This

1 The term "Open" refers to one of its major development components OpenCV, and "SURF" is the abbreviation of Speeded Up Robust Features. 
method detects significant interest points from many distributed points on input images, and it extracts regions of interest which are suitable for geometric model analysis.

\subsection{Interest Points Detection}

The implementation of OpenSURF libraries was based on an interest point detection-description scheme, first described by Bay et al. [1] in 2006. An intermediate image representation plays an important role in the improvement of SURF's performance, which is known as the "Integral Image" 11. The integral image can be computed directly from the input image by the following formula:

$$
I_{\sum}(x, y)=\sum_{i=0}^{x} \sum_{j=0}^{y} I(x, y)
$$

where $I$ is the input image, and $(x, y)$ are the $\mathrm{x}$ - and $\mathrm{y}$-coordinates of a certain pixel on the input image. So the integral image $I_{\sum}$ can be then calculated by the formula given above [4].

Figure 4 shows a sample scanned tracking card image with many interest points detected before applying the filtering function. The blue circles indicate the interest points that have black marks on a white background with a detected radius.

However, it is too difficult to define the proper regions of interest on the input image at this stage, because the representation of small species' footprints

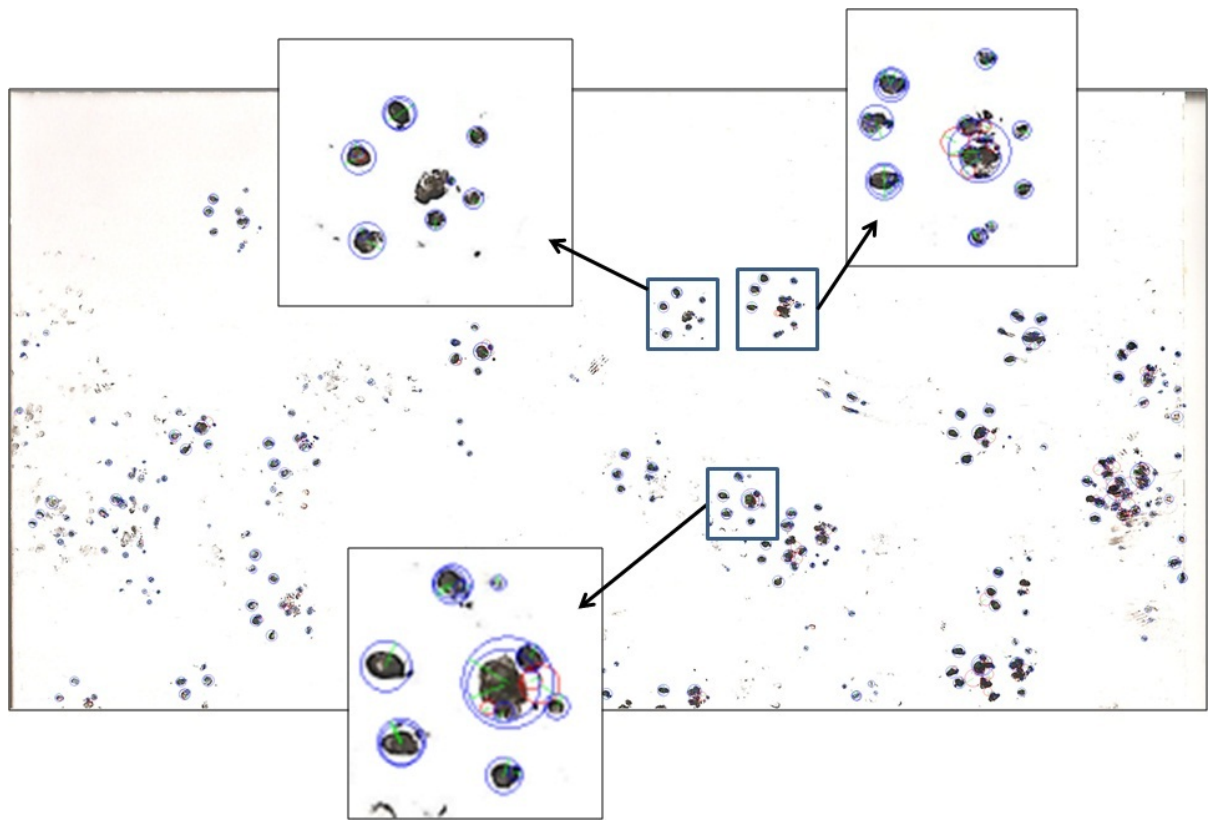

Fig. 4. Raw analysis result for a sample tracking card image 

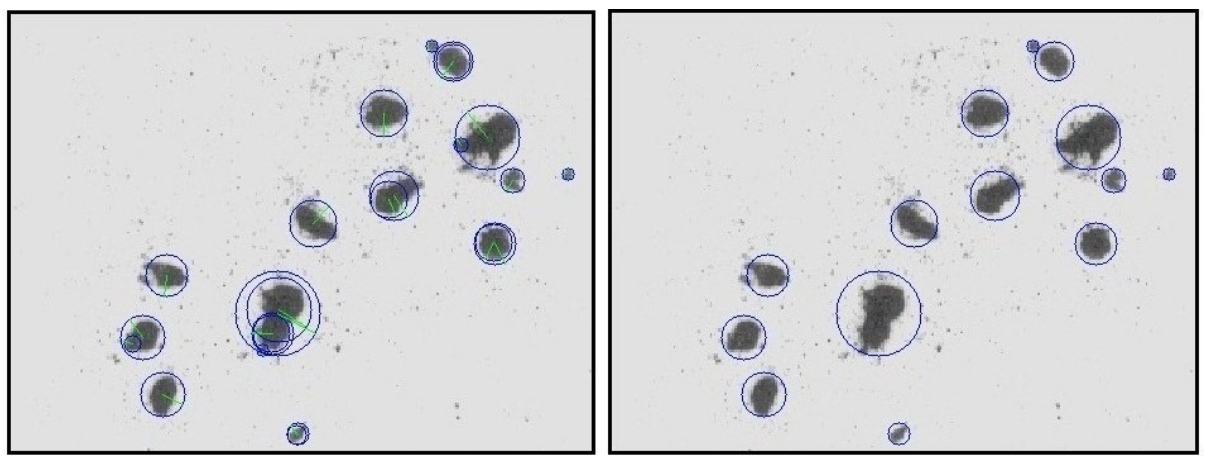

Fig. 5. Left: Detected interest points before preprocessing. Right: Identified areas of interest after preprocessing.

are commonly massively distributed, and too many interest points are detected, including many insignificant points. This certainly requires a filtering function to remove all irrelevant interest points. Therefore we implemented a rule-based filtering algorithm to remove insignificant interest points.

\subsection{Regions of Interest Extraction}

Applying this rule-based conditional filtering function, most of the insignificant or noisy interest points can be detected and removed. After applying a rule-based conditional filtering function, most of the insignificant or noisy interest points can be detected and removed. Figure 5 (Left) shows all the initially detected interest points on an input image. Figure 5 (Right) presents the experimental result after using filtering rules. Basically all the regions of interest on the input image are detected and localised correctly. For the filtering algorithm, see Figure 7.

Analysing the standard geometric models could provide us with the following organised truths of our study object's footprints:
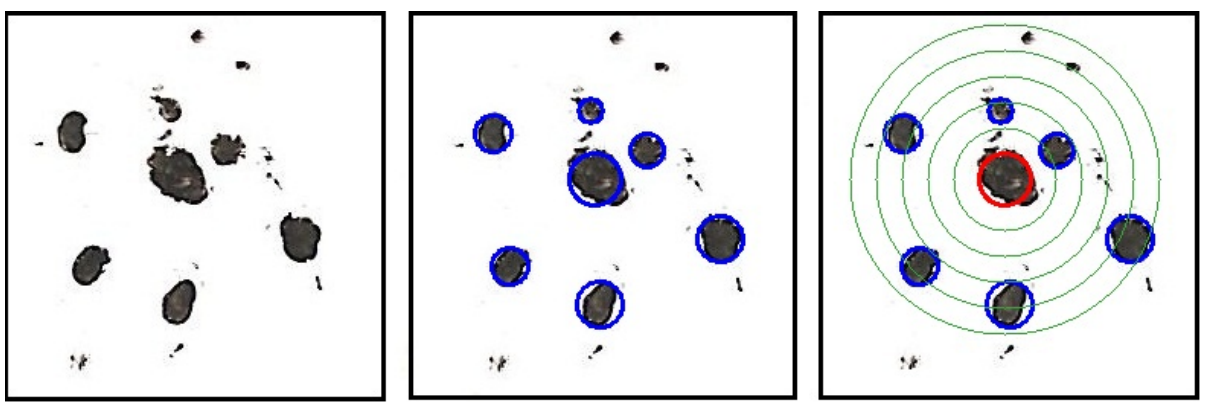

Fig. 6. Left: Original image. Middle: Interest points preprocessed by our application. Right: Central pad localisation by our application. Blue circles indicate 'area of interest'; red circle indicate 'recognised central pad'; green circles indicate distance from the centre of the central pad, each gap represents one times the central pad radius. 
1: Initialise $\mathbf{L}$; $\{$ the list of interest points on the input image

2: for (each interest point $\mathbf{I}$ in list $\mathbf{L}$ ) do

3: if (the radius of $\mathbf{I}$ is less than 6 pixels) then

4: $\quad$ Delete the interest point from list $\mathbf{L}$;

5: else

6: $\quad$ Initialise $\mathbf{V}$; $\{$ an empty interest point list $\}$

7: $\quad$ for (each interest $\mathbf{K}$ in the list $\mathbf{L}$ ) do

8: $\quad$ if (the radius of $\mathbf{K} \geq$ the radius of $\mathbf{I}$ ) then

9: $\quad$ Initialise $\mathbf{D} ;\{$ the distance between $\mathbf{I}$ and $\mathbf{K}$ \}

10: $\quad$ if (the radius of $\mathbf{K} \geq$ the radius of $\mathbf{I}+\mathbf{D}$ ) then

11:

12 :

13:

14:

15:

16:

17:

18:

19:

20:

21:

22:

23:

24:

25:

26:

27:

28:

29:

30:

31:

32:

33: if (the radius of $\mathbf{K} \geq 7 / 10$ of the radius of $\mathbf{I}$ ) then Store $\mathbf{K}$ in $\mathbf{V}$

end for

Fig. 7. Pseudo-code for the pre-processing stage

Accurate matching: a central pad normally has the largest area within the six times its radius bounded region, and there should be exactly six (for the front footprint) or seven (for the hind footprint) smaller areas of interest in that particular region.

Loose matching: a central pad normally has a radius larger than the average radius within the six times its radius bounded region, and the number of areas of interest in this particular region should be greater than or equal to four, and less than or equal to ten.

Figure [6] shows the progress of locating a possible central pad on an input image. We use green circles to indicate the distance from the centre of the central pad, which also is the centre of the possible region for a footprint. From the inside to the outside boundary, each gap between every two green circles represents the 


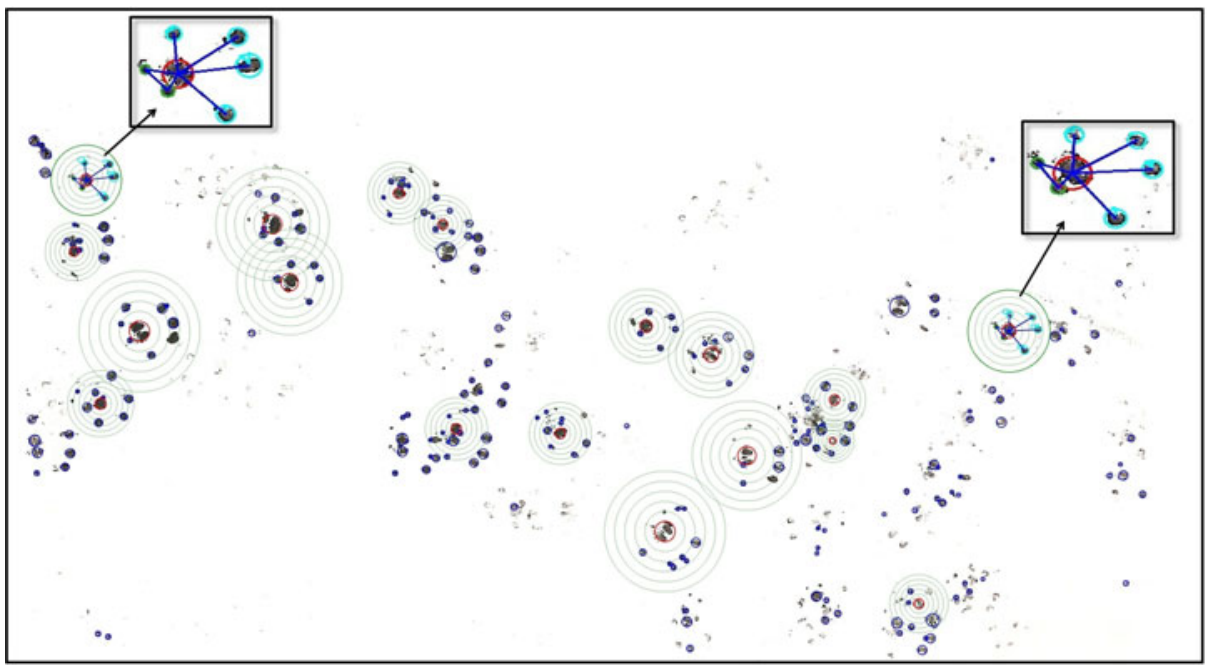

Fig. 8. Footprint detection result of an input tracking card image. Fully recognised footprints are indicated by zooming in.

length of the radius of the central pad area. The outside boundary shows the region of a possible footprint on the image. This region could be valuable when human experts decide to do manual additional analysis of the tracking card.

As the central pad can be recognised, the region of a possible footprint is located with a proper boundary, which is six times the radius of the central pad. The algorithm can then test the interest points within this range for whether their distribution matches the pre-defined model. We again defined a rule-based approach for the footprint identification and localisation. Figure 8 shows the result image after processed by the following rules:

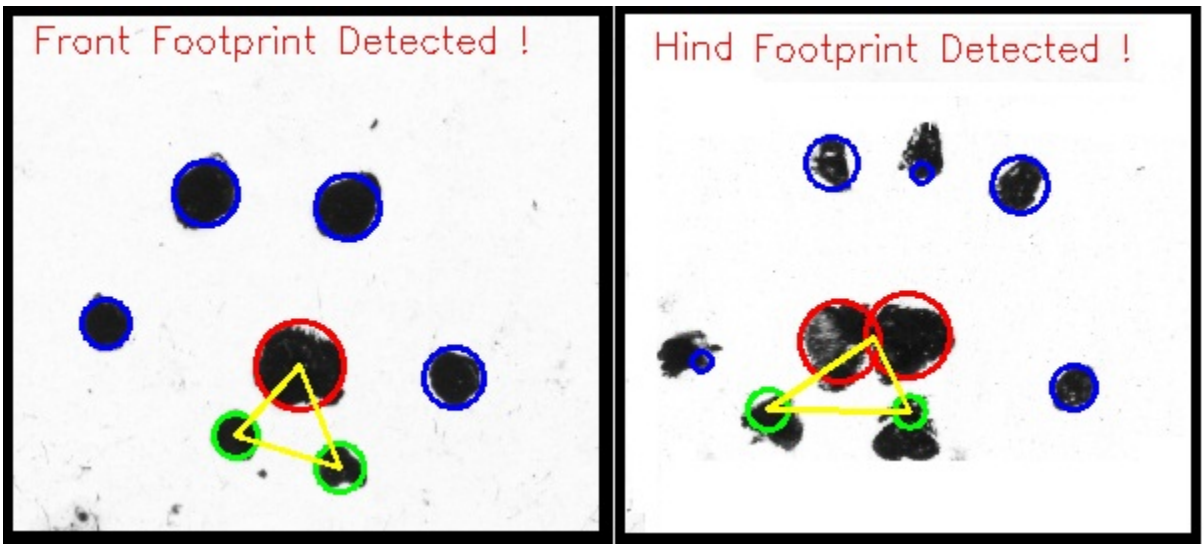

Fig. 9. Testing results for front and hind footprints 
Rule 1. Two accessorial pads should be close to the central pad, generally within the range of three times the radius of the central pad.

Rule 2. Two accessorial pads must have smaller distance to each other than their distance to other areas of interest in this particular region.

Rule 3. Two accessorial pads and the central pad can form a triangle at the back of the footprint. Each angle inside the triangle should be only smaller than or equal to $90^{\circ}$, and the sum of the three angles is exactly $180^{\circ}$.

Rule 4. A line segment can be drawn between every two toes. The longest line segment, which is the line between the left and the right outer toes, must cross the area of the central pad.

Rule 5. If the region with a recognised central pad can not completely match all the rules, it should be considered and marked as a 'possible region of a footprint' on the result image for human experts to review.

Rule 6. If the region with a recognised central pad has more than ten areas of interest within its considerable range, which is six times the radius of the central pad, the algorithm should identify this region as an 'unpredictable region', and it will refuse to do any further analysis.

Rule 7. If only one central pad is detected in this region, and also two accessorial pads and four toes are detected, the algorithm will identify it as a front footprint of our study object.

Rule 8. If two central pads are detected in this region, and also two accessorial pads and five toes are detected, the algorithm will identify it as a hind footprint of our study object.

Since normally every two nearby toes have certain angles in between, a statistical analysis was used to find out the angles between every two nodes of the footprint samples. The corresponding statistical analysis of those angles provides us the following additional classification rules:

Front or hind footprint classification: for front footprints, the average value for angle $\angle \mathrm{T}_{2} \mathrm{CT}_{3}$ is $46.2^{\circ}$ in the range from $43.6^{\circ}$ to $48.9^{\circ}$; for hind footprints, the average value for angle $\angle \mathrm{T}_{2} \mathrm{CT}_{3}$ is $56.1^{\circ}$ in the range from $53.2^{\circ}$ to $59.8^{\circ}$. There is a clear difference between the two ranges.

Left or right footprint classification: if angle $\angle \mathrm{A}_{1} \mathrm{CT}_{1}$ is less than angle $\angle \mathrm{T}_{4} \mathrm{CA}_{2}$, then this is a left footprint; otherwise, this is a right footprint.

Figure 9 presents two fully recognised footprints of our study object with expected classification results. In this case, the algorithm counts the number of central pads detected, a front footprint should have one central pad whereas a hind footprint should have two. Also the number of toes are different, a front footprint should have four toes, and a hind footprint should have five.

\subsection{Geometric Feature Extraction}

By understanding the standard geometric models of our study object's footprints, we could gather useful information for relations among nodes within one region of interest, such as angles, areas, or distances. These geometric features can be generally found by footprints which are left by small species. Analysing 
the detected features statistically can certainly provide strong proof of footprint localisation and classification results. We extract geometric features from associated nodes in the regions of interest. Data collection is only as valid as the feature extraction, and is considered an important objective of our application.

In order to collect statistical analysis data for classifying similar species (e.g. rats and mice), we have to sort the accessorial pads and toes in a certain order (e.g. sort them clockwise). The application would collect (1) radius of each node; (2) distance between each node and central pad; (3) the area of the triangle (formed by central pad and its accessorial pads); (4) internal angles of that triangle, etc. These statistic data will be used to find definitive differences for distinguishing morphologically similar species, such as rats and mice.

\subsection{Directional Scale Vector}

Every footprint has a relative direction. The footprint directions are indispensable when we try to find a single track of an individual mammal. In our approach, we use a $2 \mathrm{D}$ vector $\mathbf{v}_{d s}$ to present the relative direction of a given footprint, it is called directional scale. Based on the extracted geometric regions and their centroids, the directional scale vector $\mathbf{v}_{d s}=\left(x_{s}, y_{s}\right)$ can be calculated by the following formula:

$$
x_{s}=\left(\sum_{i=1}^{n_{r}} x_{i}\right)-n_{r} \cdot x_{c p} \quad \text { and } \quad y_{s}=\left(\sum_{i=1}^{n_{r}} y_{i}\right)-n_{r} \cdot y_{c p}
$$

Here, $n_{r}$ is the number of regions (i.e. toes and accessorial pads of the given footprint, not counting the central pad), $x_{c p}$ and $y_{c p}$ are the $x$ - and $y$-coordinates of the centroid of the central pad, and $x_{i}$ and $y_{i}$ are the coordinates of the centroid of region $i$. This vector can precisely indicate the relative directions of any footprints. By picking up those footprints with similar direction scale values, we can then directly indicate which path on the tracking card the current footprint belongs to.

\section{Experimental Test}

In order to systematically test the accuracy of our algorithm, we test accuracy on two datasets of mice tracked on cards. The first study is of introduced house mice from New Zealand and the second study of hazel dormice from the United Kingdom. The combined image data set has been divided into three data groups (as shown in Figure 10). We recorded the experimental data during application of the track recognition algorithm. The experimental results are reported by tracking card for the three different image data groups in four different categories, where each category represents a classification related to regions of interest on a card. Thus a single card with multiple regions of interest may fall in more than one of the following categories explained below, depending on the number of regions identified: 


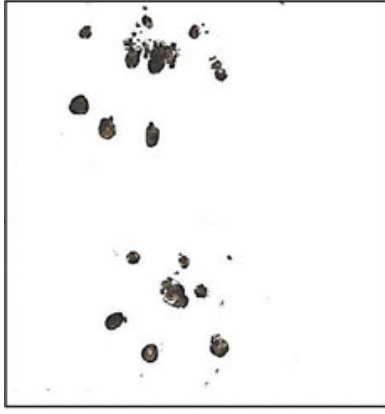

(Group 1)

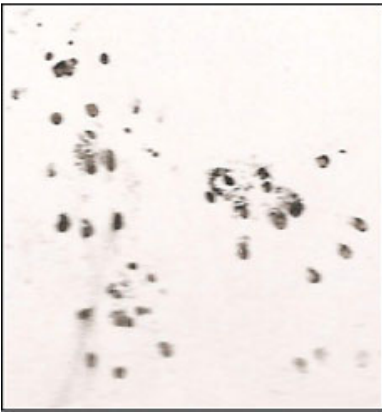

(Group 2)

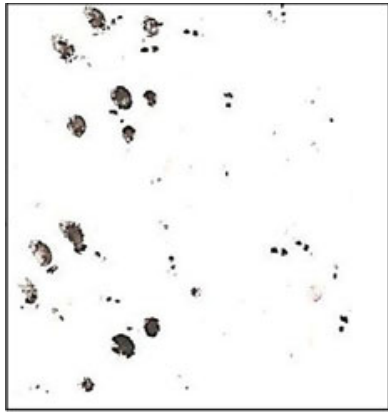

(Group 3)

Fig. 10. The preview images of the three image data groups. (Group 1) clear foreground and background; (Group 2) dim foreground and background; (Group 3) unexpected species involved (here an invertebrate).

Sensitive Matches: The number of cards containing an identified footprint fully matching the pre-defined rules in the algorithm (i.e. confirmed species presence).

Loose matches (True): The number of cards containing a region of interest loosely identified as a possible region of footprints, where it is truly one footprint (i.e. footprint identified).

Loose matches (False): The number of cards containing a region of interest loosely identified as a possible region of footprints, but it is not a footprint (i.e. mis-identification).

Did not detect print: The number of cards where no footprints are detected by the algorithm (i.e. species absence).

The experimental result (as shown in Table 1) indicates that the algorithm has a fairly high success rate for sensitive footprint identification and loose-condition matches for images with clear prints and clean background.

The accuracy for dim background and foreground images is lower than the results for images from clear background and foreground images. In addition, the accuracy for tracking cards with tracks from unexpected species

Table 1. The experimental statistical analysis for the algorithm accuracy evaluation. "Sensitive matches" indicates rate of best matched footprints. Correctly or incorrectly detected possible footprints are assigned as "loose matches (true)" or "loose matches (false)". "Did not detect print" records no footprints detected for a card.

\begin{tabular}{lccc}
\hline \hline & \multicolumn{3}{c}{ Percentage of Detection Accuracy } \\
\cline { 2 - 4 } Classification & Group 1 (72 cards) & Group 2 (42 cards) & Group 3 (22 cards) \\
\hline Sensitive matches & $77.8 \%$ & $61.9 \%$ & $68.2 \%$ \\
Loose matches (True) & $85.7 \%$ & $68.3 \%$ & $80.7 \%$ \\
Loose matches (False) & $14.3 \%$ & $31.7 \%$ & $19.3 \%$ \\
Did not detect print & $1.4 \%$ & $9.5 \%$ & $9.1 \%$ \\
\hline \hline
\end{tabular}


(e.g. invertebrates) is surprisingly good; the reason being that the track recognition algorithm has a filtering function that filters out all the tracks with very small regions, which might be left by unknown species other than our object of study (e.g. invertebrate tracks).

\section{Conclusions}

We propose a method for locating and classifying footprints of small species on scanned tracking card images by three major steps: (1) extracting regions of interest; (2) further analysis with "rule-based conditional filtering"; (3) extracting footprint "geometric features". In addition, our method can provide useful results for finding footprints which belong to the same path with a $2 \mathrm{D}$ vector called "direction scale".

Comparing with some previous studies 6 6 801012] in this research field, we propose two new ideas for this algorithm:

A footprint could be identified either "fully matched" or "partially matched", which depends on the degree of matching the pre-defined rules. Due to the sparse amount of information provided by the detected interest points, rulebased identification processes could be a key to the shortage of information. Moreover, rule-based identification could allow developers to add a new rule or modify the existing rules. This provides a greater extensibility to this algorithm.

Footprint geometric models could provide precise mathematical relationships among nodes of the standard footprint for any target species. In practical implementations, numbers, equations and formulas are always considered useful information for footprint identification (e.g. [10] ).

The experimental results provide positive feedback on the accuracy of this algorithm; if the image cards have clear prints and clean background, $85.7 \%$ of them can be detected as "loosely matched" by the pre-defined rules of this algorithm.

Acknowledgements. We are grateful to Jamie MacKay from the University of Auckland and Cheryl Mills from the University of Exeter for access to tracking cards ( $n=50$ and $n=86$ cards, respectively).

\section{References}

1. Bay, H., Tuytelaars, T., Van Gool, L.: SURF: Speeded up Robust Features. In: Leonardis, A., Bischof, H., Pinz, A. (eds.) ECCV 2006. LNCS, vol. 3951, pp. 404417. Springer, Heidelberg (2006)

2. Blackwell, G.L., Potter, M.A., McLennan, J.A.: Rodent density indices from tracking tunnels, snap-traps and Fenn traps: do they tell the same story? New Zealand Journal of Ecology 26, 43-51 (2002)

3. Brown, K., Moller, H., Innes, J., Alterio, N.: Calibration of tunnel tracking rates to estimate relative abundances of ship rats and mice in a New Zealand forest. New Zealand Journal of Ecology 20, 271-275 (1996) 
4. Evans, C.: Notes on the OpenSURF library. University of Bristol (January 2009), Www.cs.bris.ac.uk/Publications/Papers/2000970.pdf (last accessed on July 15, 2011)

5. Gaston, K.J., O'Neill, M.A.: Automated species identification: why not? Philosophical Transactions of the Royal Society B 359, 655-667 (2004)

6. Lowdon, I.M.R., Seaber, A.V., Urbanlak, J.R.: An improved method of recording rat tracks for measurement of the sciatic functional index of deMedinaceli. Journal of Neuroscience Methods 24, 279-281 (1988)

7. MacKenzie, D.I., Nichols, J.D., Lachman, G.B., Droege, S., Royle, J.A., Langtimm, C.A.: Estimating site occupancy rates when detection probabilities are less than one. Ecology 83, 2248-2255 (2002)

8. Mayo, M., Watson, A.T.: Automatic species identification of live moths. Knowledge-Based Systems 20, 195-202 (2007)

9. Medinaceli, L.D., Freed, W.J., Wyatt, R.J.: An index of the functional condition of rat sciatic nerve based on measurements made from walking tracks. Experimental Neurology 77, 634-643 (1982)

10. Russell, J.C., Hasler, N., Klette, R., Rosenhahn, B.: Automatic track recognition of footprints for identifying cryptic species. Ecology 90, 2007-2013 (2009)

11. Viola, P., Jones, M.: Rapid object detection using a boosted cascade of simple features. In: Conference on Computer Vision and Pattern Recognition, vol. 1, pp. $511-518(2001)$

12. Watts, C.H., Thornburrow, D., Green, C.J., Agnew, W.R.: Tracking tunnels: a novel method for detecting a threatened New Zealand giant Weta. New Zealand Journal of Ecology 32, 92-97 (2008) 